\title{
Anti-Tumor Activity of Genetically Redirected T Cells Against Orthotopic Kidney Cancer in Mice
}

\author{
Jennifer A. Westwood ${ }^{1}$, Preethi Mayura-Guru ${ }^{1}$, William K Murray ${ }^{2}$, Hollie J. Pegram ${ }^{1}$, \\ Sally M. Amos ${ }^{1}$, Carol L. Van Puyenbroek ${ }^{1}$, Rachel Cameron ${ }^{1}$, Mark J. Smyth ${ }^{1,3}$, \\ Yuekang Xu ${ }^{1,3}$, Phillip K. Darcy ${ }^{1,3}$ and Michael H. Kershaw ${ }^{1,3, *}$ \\ ${ }^{I}$ Cancer Immunology Research Program, Peter MacCallum Cancer Centre, Melboume, Australia \\ ${ }^{2}$ Department of Pathology, Peter MacCallum Cancer Centre, Melbourne, Australia \\ ${ }^{3}$ Department of Pathology, University of Melbourne, Australia
}

\begin{abstract}
In order to test the effectiveness of adoptive immunotherapy against renal cell carcinoma (RCC) in a mouse model relevant to human disease, we first generated two murine renal cell carcinoma cell lines expressing the human tumor-associated antigen erbB2 by genetic modification of the Renca and SIRCC cell lines. When injected into the kidneys of mice, these cell lines rapidly formed a primary kidney tumor that metastasized to lung, liver and various peritoneal locations. Tumor-specific mouse $\mathrm{T}$ cells were generated by genetic modification of splenic $\mathrm{T}$ cells in vitro. The transgene encoded a chimeric receptor (T-body) specific for erbB2, in which a single-chain (scFv) anti-erbB2 antibody was linked to $\mathrm{T}$ cell activation and costimulatory domains (scFv-erbB2-CD28-zeta), to enable tumor recognition in a major histocompatibility complex-independent manner. Gene-modified $\mathrm{T}$ cells expressed the T-body on their surface, and could respond specifically against erbB2-expressing tumor cells as demonstrated by secretion of interferon-gamma. Adoptive transfer of T-body-expressing $\mathrm{T}$ cells could lead to inhibition of primary tumor and metastases. Systemic delivery of these gene-modified $\mathrm{T}$ cells led to complete eradication of disease in a proportion of mice, and this was associated with peri-vascular tissue inflammation composed of activated lymphoid cells, with macrophages and lesser numbers of eosinophils and neutrophils. This study demonstrated, for the first time, the efficacy of genetically redirected $\mathrm{T}$ cells against orthotopic renal cell carcinoma in mice.
\end{abstract}

Keywords: Gene therapy, Adoptive immunotherapy, Genetic vectors, Renal cell carcinoma, T lymphocytes.

\section{INTRODUCTION}

Kidney cancer can be responsive to immunotherapy. This is most clear from the use of cytokines such as interleukin-2, which can achieve a $20 \%$ response rate in patients with renal cell carcinoma, with approximately $6 \%$ complete responses, some of which are long lasting [1]. Similarly, a combination of IFN- $\alpha$ and chemotherapy can benefit patients; with response rates similar to those achieved using IL-2 [2]. In addition, allogeneic hematopoietic stem cell transplant can also impact on RCC in patients through a graft versus tumor effect resulting in an overall response rate of approximately $25 \%$ [3]. While these approaches serve to highlight the potential of the immune system to impact on kidney cancer, complete durable responses remain all too few, and there is a clear need for improvements to immunotherapeutic approaches for these diseases.

It is becoming evident that $T$ cells specifically reactive with RCC can be isolated, and a number of approaches have been demonstrated to stimulate specific $\mathrm{T}$ cells in a proportion

*Address correspondence to this author at the Cancer Immunology Research Program, Peter MacCallum Cancer Centre, Melboume, Australia;

Tel: (613) 9656 1177; Fax: (613) 9656 1411;

E-mail: michael.kershaw@petermac.org of patients [4]. However, it is not currently possible to generate effective RCC-reactive $\mathrm{T}$ cells from the endogenous immune repertoire of most patients.

An alternate method of generating tumor-reactive T cells is through the genetic modification of peripheral blood lymphocytes using genes encoding cell surface receptors that are able to bind to tumor antigens and transmit activation signals, thereby enabling a response against tumor cells. Genes currently being assessed encode either alpha and beta chains of the T cell receptor (TCR), or chimeric single-chain antibody (scFv)-based receptors (T-bodies). In this way, large numbers of gene-modified autologous $\mathrm{T}$ cells can be generated in vitro and administered to patients as an intravenous infusion.

A range of chimeric scFv T-bodies have been developed targeting a wide variety of tumor-associated antigens (TAA) including erbB2 [5], folate-binding protein (FBP) [6], CD19 [7, 8], prostate-specific membrane antigen (PSMA) [9], IL-13 receptor [10], carcinoembryonic antigen (CEA) [11] and Lewis-Y [12]. These antigens are expressed on a range of malignancies including lymphoma, glioblastoma and carcinomas of the breast, ovary, prostate and colon.

Given the exciting potential of adoptive immunotherapy to impact on advanced cancers (chiefly melanoma) it is 
highly desirable to extend this approach to kidney cancer. Indeed, tumor-associated antigens have been identified for urological cancers including carbonic anhydrase IX (CAIX) [13] and erbB2 [14], and T-bodies specific for these antigens exist that can endow $\mathrm{T}$ cells with reactivity against kidney tumor cells in vitro [15], but no studies have investigated the anti-tumor effect of adoptively transferred genetically redirected $\mathrm{T}$ cells against kidney cancer in mice. Reasoning that an appropriate mouse model would provide the opportunity to more stringently test and optimize this approach against kidney cancers, we generated murine renal cell carcinoma cell lines expressing human erbB2, and sought to establish an orthotopic model of kidney cancer using these cell lines in mice. Here we describe this model and determine the effectiveness of gene-redirected $\mathrm{T}$ cells against primary and spontaneously metastatic kidney cancer.

\section{MATERIALS AND METHODS}

\section{Cell Lines, Mice and Antibodies}

The mouse renal cell carcinoma cell line, SIRCC, was a chemically-induced renal cell carcinoma of BALB/c mice, kindly provided by Dr. R.H. Wiltrout, Frederick, MD. Renca is a renal cell carcinoma cell line [16]. The erbB2-expressing cell lines Renca-erbB2 and SIRCC-erbB2, were generated by transduction with a retroviral vector (MSCV) encoding human erbB2. Tumor cell lines were maintained at $37^{\circ} \mathrm{C}$ and $5 \% \mathrm{CO}_{2}$ in RPMI medium, supplemented with $10 \%$ heatinactivated fetal calf serum, $2 \mathrm{mM}$ glutamine, $100 \mathrm{U} / \mathrm{ml}$ penicillin, and $100 \mu \mathrm{g} / \mathrm{ml}$ streptomycin.

The murine ecotropic retroviral producer cell line $\mathrm{GP}+\mathrm{E} 86$-erbB2 was generated by transfection of GP+E86 (ATCC) with the retroviral expression plasmid pLXSNscFv- $\alpha$-erbB2-CD28- $\zeta$ as previously described [17] and was maintained in DMEM medium (Gibco, Grand Island, NY, USA), supplemented as above. Mouse T cells were cultured in supplemented RPMI, containing $100 \mathrm{IU} / \mathrm{ml}$ human recombinant interleukin-2 (rh-IL-2) (Biological Resources Branch Preclinical Repository, NCI, Frederick, MD).

BALB/c and SCID mice were purchased from The Walter and Eliza Hall Institute of Medical Research, Melbourne, Australia, and from Animal Resource Centre, Perth, Western Australia. Mice of 6 to 12 weeks of age were used in all experiments, and experiments were performed according to The Peter MacCallum Cancer Centre Animal Experimentation Ethics Committee guidelines.

Antibodies used in this study were a mouse monoclonal anti-human erbB2 (clone 9G6.10; NeoMarkers, Fremont, $\mathrm{CA}$ ), and a mouse mAb specific for a 9-aa epitope of human c-myc that was incorporated into the T-body design (clone 9B11; Cell Signaling Technology, Beverly, MA).

\section{Generation of erbB-2-Reactive T Cells}

Mouse $\mathrm{T}$ cells reactive with erbB2 were generated by retroviral transduction using an anti-erbB2 chimeric receptor (T-body) as described previously [18]. Briefly, T cells were enriched from mouse spleens using nylon wool and $1 \times 10^{7}$ were plated on $10 \mathrm{~cm}$ tissue culture plates containing $5 \times 10^{5}$ $\mathrm{GP}+\mathrm{E} 86$ retroviral producer cells, in the presence of $5 \mu \mathrm{g} / \mathrm{ml}$ phytohemagglutinin and $4 \mu \mathrm{g} / \mathrm{ml}$ polybrene. IL-2, $100 \mathrm{IU} / \mathrm{ml}$, was included in the cultures. After 72 hours, T cells were separated from the adherent producer cells, washed and replated for a further 24 hours with IL-2, before harvest for experiments. The T-body is composed of a single-chain antierbB2 antibody linked to the transmembrane and cytoplasmic domains of CD28 and the signaling domains of TCR- $\zeta$ (scFv-erbB2-CD28-zeta), which has previously been demonstrated to be capable of inducing regression of breast cancer in mice [18]. Control $\mathrm{T}$ cells were generated by either a mock transduction method in which splenocytes were incubated in culture dishes using all procedures as above but in the absence of producer cells, or using the empty LXSN vector where indicated.

\section{Cytokine Secretion: ELISA}

Cytokine secretion in response to tumors was determined by coculture of $1 \times 10^{6}$ erbB2-specific T cells with $5 \times 10^{5}$ tumor cells, in 12-well tissue culture plates in a final volume of $1 \mathrm{ml}$ supplemented RPMI with no IL-2. Following overnight incubation of plates at $37^{\circ} \mathrm{C} / 5 \% \mathrm{CO}_{2}$, supernatant was harvested and assayed for IFN- $\gamma$ in duplicate wells of 96-well flat-bottom plates, using enzyme-linked immunosorbent assays (ELISA), performed according to manufacturer's instructions (BD Biosciences).

\section{Tumor Growth in Mice}

Groups of five SCID mice were inoculated with $3 \times 10^{4}$ Renca or $1 \times 10^{5}$ SIRCC tumor cells sub-capsular in the kidney. Recipient mice were anaesthetized with an i.p. injection of Ketalar (200 mg/kg, West Ryde, Australia) plus Xylazine Hydrochloride $(10 \mathrm{mg} / \mathrm{kg}$, Troy Laboratories, Smithfield, NSW, Australia). One kidney was exteriorized via flank incision. Tumor cells $\left(3 \times 10^{4}\right)$ in a 50:50 Matrigel (BD Biosciences):PBS mix were injected in a $50 \mu \mathrm{l}$ volume using a Hamilton syringe (Hamilton Co. Reno, NV) fitted with a 27 gauge needle. The kidney was then returned, the peritoneum sutured, and the incision closed using surgical clips (which were removed after 10 days). Some groups received $0.4 \mathrm{ml}$ saline containing $1 \times 10^{7}$ anti-erbB2 $\mathrm{T}$ cells or mocktransduced $\mathrm{T}$ cells by intravenous injection 24 and 48 hours after tumor inoculation for the Renca tumor model, or a single injection of $1 \times 10^{7} \mathrm{~T}$ cells on day 4 after tumor injection for the SIRCC model. Tumor progression was determined by survival of mice, which was defined as time until mice became moribund, at which point they were euthanized.

\section{RESULTS}

\section{Generation of Murine RCC Cells Expressing erbB2}

The cDNA encoding human erbB2, cloned into the retroviral expression vector MSCV, was used to transduce the mouse RCC cell lines, Renca and SIRCC. Cell lines enriched for erbB2-expressing cells were derived using cloning by limiting dilution, analysis of erbB2 expression by flow cytometry, and pooling of over 6 clones. Expression of erbB2 was demonstrated using flow cytometry following staining with erbB2-specific monoclonal antibody (Fig. 1). The level of erbB2 expression was typical of many naturally occurring carcinomas. This provided RCC cell lines expressing erbB2, and the non-expressing parental cell lines for use to determine the role of erbB2 targeting in immune-mediated antitumor effects. 


\section{Generation of erbB2-Reactive T Cells}

Following coculture of $\mathrm{T}$ cell-enriched mouse splenocytes with T-body retroviral producer cells, non-adherent lymphocytes were separated from the adherent producer cells and analyzed by flow cytometry. Expression of the antierbB2 T-body was demonstrated by the increase in fluorescence following staining with a monoclonal antibody specific for the c-myc epitope incorporated into the T-body (Fig. 2a). Specificity of staining was confirmed by the lack of staining of mock-transduced $\mathrm{T}$ cells (Fig. 2b). Approximately $30-50 \%$ of $\mathrm{T}$ cells expressed the T-body following transduction. Typically, cultures were largely $\mathrm{CD}^{+}$(range $70-85 \%$ ) with a lower proportion of $\mathrm{CD}^{+} \mathrm{T}$ cells (range 10$20 \%$ ) (Fig. 2c). Minor percentages of $\mathrm{CD} 4^{+} \mathrm{CD} 8^{+}$double positive and double negative cells were also present.

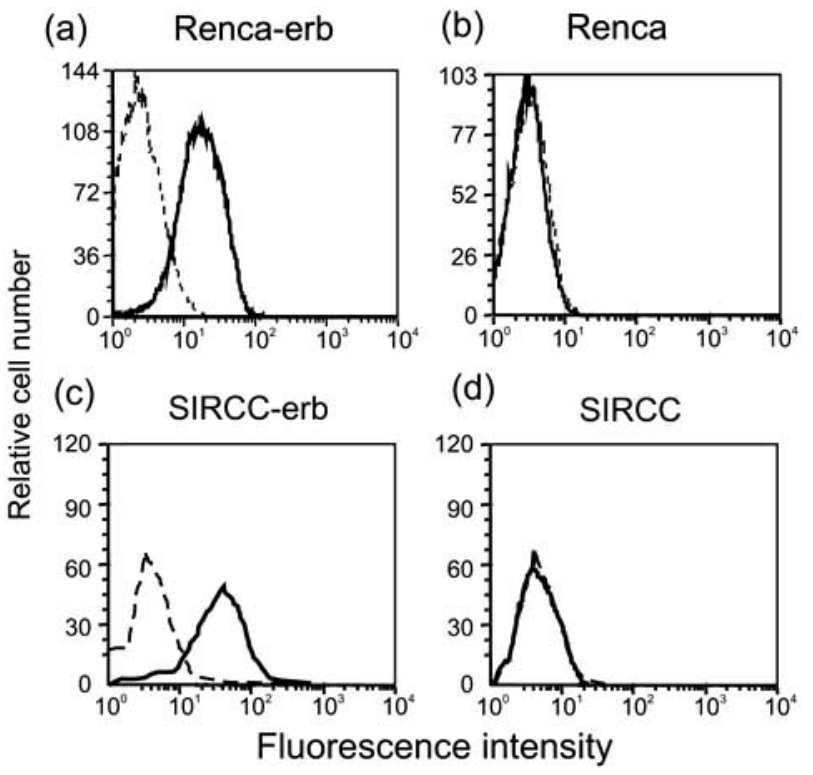

Fig. (1). Human erbB2 is expressed by transduced Renca cells. Following transduction of Renca and SIRCC, the level of expression of erbB2 on this cell line was determined using flow cytometry. (a) and (c) ErbB-2-transduced cells Renca and SIRCC respectively stained with an anti-erbB2-specific monoclonal antibody (solid line) compared to staining with control antibody (broken line). (b) and (d) Parental Renca and SIRCC cell lines respectively stained with anti-erbB-2 (solid line) or control antibody (broken line). This analysis is representative of more than five experiments performed throughout the study.

\section{Anti-erbB2 T Cells Respond Against Tumor Cells}

Having demonstrated that the anti-erbB2 T-body could be expressed on the surface of mouse $\mathrm{T}$ cells, it was next important to determine if the $\mathrm{T}$ cells expressing the antierbB2 T-body were reactive against erbB2 $2^{+}$tumor cells. This was done by measuring the secretion of IFN- $\gamma$ by $\mathrm{T}$ cells after coculture with various target cells. $\mathrm{T}$ cells transduced with the anti-erbB2 T-body were demonstrated to secrete IFN- $\gamma$ in response to erbB $2^{+}$tumor cells, but not in response to erbB2 ${ }^{-}$tumor cells (Fig. 3). The requirement for chimeric anti-erbB2 T-body expression for this response was apparent from the lack of activity of control mock-transduced $\mathrm{T}$ cells against erbB2 $2^{+}$tumor cells.

\section{Anti-erbB2 T Cells Inhibit Tumor Growth}

Having demonstrated the ability of the anti-erbB2 $\mathrm{T}$ cells to specifically recognize and secrete cytokine against RencaerbB2 cells in vitro, we next sought to determine if they could impact on tumor growth in vivo.

(a) Anti-erb T cells

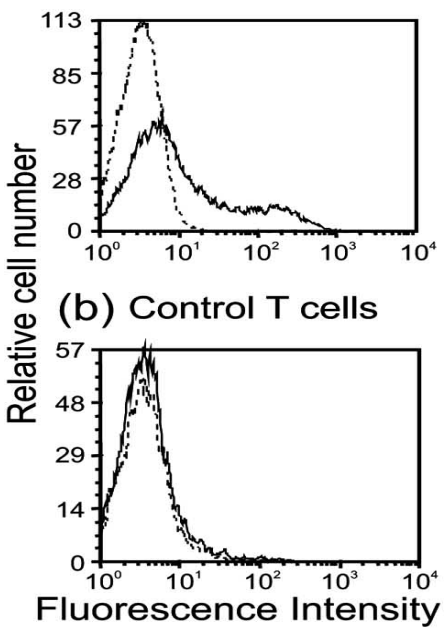

(c) Anti-erb T cells

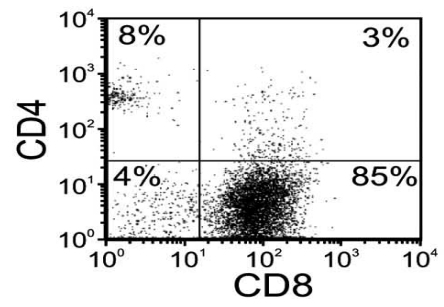

Fig. (2). Expression of anti-erbB2 T-body in mouse $T$ cells. Following transduction of mouse spleen-derived $\mathrm{T}$ cells with retrovirus encoding the chimeric anti-erbB2 T-body, cells were stained with a monoclonal antibody specific for a human c-myc epitope included in the T-body. Cell surface expression of the chimeric Tbody was apparent when stained with anti-c-myc (solid line) compared to control antibody (broken line) (a). Mock-transduced $\mathrm{T}$ cells did not express T-body (b). Anti-erbB2 T cells were composed of largely $\mathrm{CD}^{+}$(range $70-85 \%$ ) with a lower proportion of $\mathrm{CD} 4^{+} \mathrm{T}$ cells (range 10-20\%) (c). This experiment is representative of more than five experiments.

BALB/c-SCID mice were inoculated subcapsular into the kidney with the Renca or Renca-erbB2 cell lines, followed 24 and 48 hours later by intravenous injection of $1 \times 10^{7}$ anti-erbB2 $\mathrm{T}$ cells, control $\mathrm{T}$ cells or no $\mathrm{T}$ cells. Renca and Renca-erbB2 tumor cells grew at similar rates in non-treated mice (Fig. 4). Renca-bearing mice treated with anti-erbB2 $\mathrm{T}$ cells died over a similar time frame as non-treated mice. However, survival of mice bearing Renca-erbB2 tumors was significantly enhanced by anti-erbB2 T cells. Indeed, a proportion of treated mice survived long-term suggesting that tumors had been eradicated. The anti-tumor effect was dependent on transduction of $\mathrm{T}$ cells with the antierbB2 receptor since control $\mathrm{T}$ cells impacted to a much less degree on survival. Similarly, the effect was specific for the erbB2 antigen since growth of Renca tumors lacking erbB2 expression was largely unaffected by transfer of anti-erbB2 T cells. 


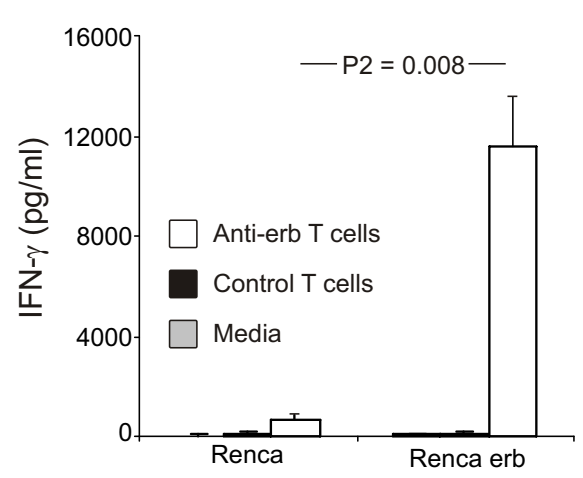

Fig (3). Anti-erbB2 $T$ cells secrete IFN- $\gamma$ in response erbB2 ${ }^{+}$ tumor cells. T cells were transduced with the anti-erbB2 T-body or mock transduced, and cultured with tumor cells with or without erbB2 expression. After overnight incubation, IFN- $\gamma$ in the supernatant was quantitated using ELISA. Anti-erbB2 $\mathrm{T}$ cells secreted IFN- $\gamma$ specifically in response to erbB2 ${ }^{+}$and not erbB2 ${ }^{-}$ tumor cells $\left(\mathrm{P}_{2}=0.008\right.$, Mann-Whitney test). Mock-transduced $\mathrm{T}$ cells were non-responsive against erbB $2^{+}$tumor cells, thereby demonstrating the requirement for the anti-erbB2 T-body for cytokine secretion. (Error bars $=$ SD from 5 experiments).

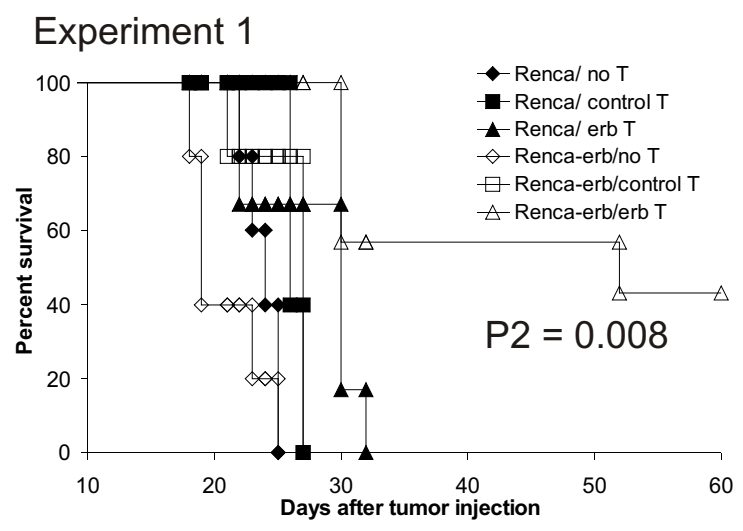

\section{Experiment 2}

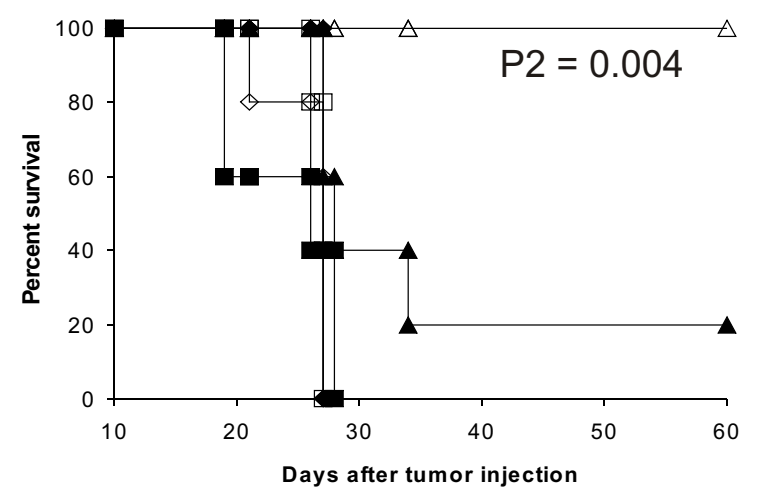

Fig (4). Anti-erbB2 T cells enhance the survival of Renca tumorbearing mice. BALB/c-SCID mice were injected beneath the kidney capsule with $3 \times 10^{4}$ cells of either Renca or Renca-erbB2, followed on days 1 and 2 with i.v. injection of $1 \times 10^{7} \mathrm{~T}$ cells transduced with anti-erbB2 T-body or mock-transduced. Other groups of mice remained non-treated as controls. Mice receiving anti-erbB2 $\mathrm{T}$ cells survived significantly longer than all other groups of mice. Results of two experiments are shown. $\mathrm{P}$ values compare mice bearing Renca-erbB2 tumors receiving anti-erbB2 $\mathrm{T}$ cells with mice bearing Renca tumors receiving anti-erbB2 $\mathrm{T}$ cells. Two sided Fisher's exact test using Log-Rank and Wilcoxin analysis.
To provide further confirmation of the anti-tumor abilities of adoptively transferred $\mathrm{T}$ cells, we performed a further experiment using the SIRCC-erbB2 cell line according to the same protocol described for Renca. Again, transfer of erbB2specific $T$ cells significantly reduced the tumor burden of mice (Fig. 5). A significant reduction was observed in primary kidney tumor size, lung metastases and abdominal metastases at day 17 following tumor inoculation. No significant decrease in tumor burden was observed against the parental erbB2-negative cell line. Interestingly, some antitumor activity was afforded by control (empty vectortransduced) $\mathrm{T}$ cells which trended towards significance with respect to reduction in metastases when compared to nontreated tumor-bearing mice (Fig. 5).

(a)

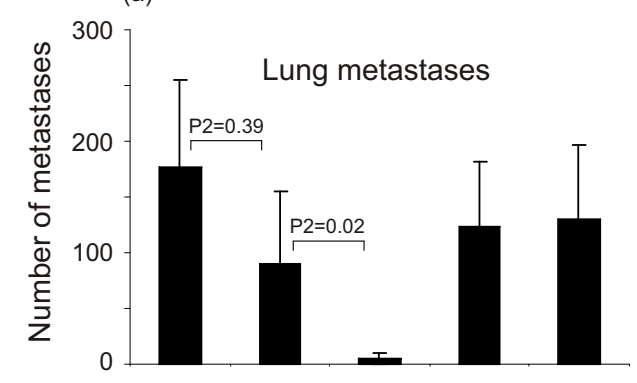

(b)

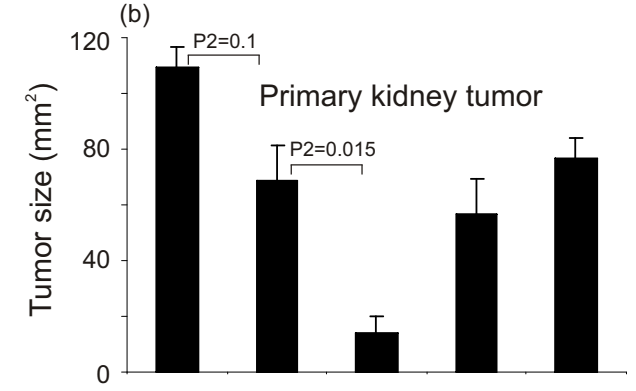

(c)

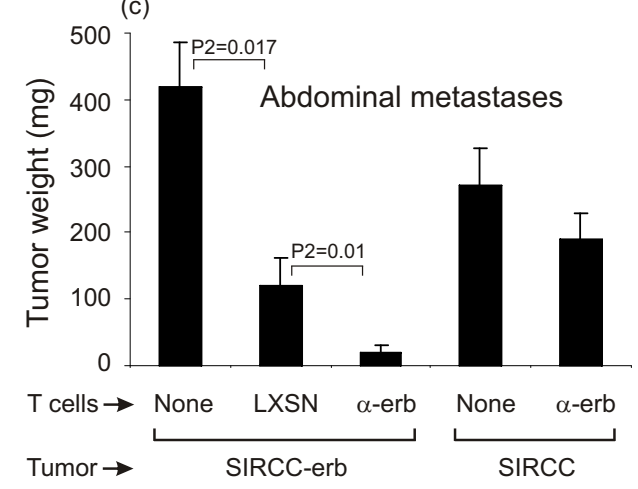

Fig (5). Anti-erbB2 $T$ cells decrease the burden of primary tumor and metastases in SIRCC tumor bearing mice. BALB/cSCID mice were injected beneath the kidney capsule with $1 \times 10^{5}$ cells of either SIRCC or SIRCC-erbB2, followed on day 4 with i.v. injection of $1 \times 10^{7} \mathrm{~T}$ cells transduced with either anti-erbB2 $\mathrm{T}$ body or empty LXSN vector. Other groups of mice remained nontreated as controls. On day 25 after tumor inoculation, mice were euthanized and tissues taken. Lung metastases were enumerated (a), primary kidney tumor was measured using calipers (b), and abdominal metastases were collected and weighed (c). Anti-erbB2 T cells significantly inhibited tumor growth compared to all other groups $(\mathrm{P} 2<0.05)$. LXSN-transduced $\mathrm{T}$ cells effected a modest inhibition of tumor growth, but only reached significance against abdominal metastases compared to non-treated mice. Statistics determined using Mann-Whitney test. 


\section{Characterization of Tumors}

In order to gain insight into disease progression in the presence or absence of specific treatment in this model, some mice were culled on day 21 after tumor inoculation and necropsy performed. Non-treated mice or those receiving control $\mathrm{T}$ cells had large primary tumors, often replacing the entire kidney, that erupted through the kidney capsule to form large tumor deposits attached to the kidney and at various peritoneal locations (Fig. 6). Lung metastases were also present in the majority of these mice, and a proportion of these mice also had liver metastases. In contrast, mice receiving anti-erbB2 $\mathrm{T}$ cells often had no evidence of disease, or had a much reduced tumor burden, often without primary disease or metastases (Fig. 6). Metastases to contralateral kidney were not seen in any mice.
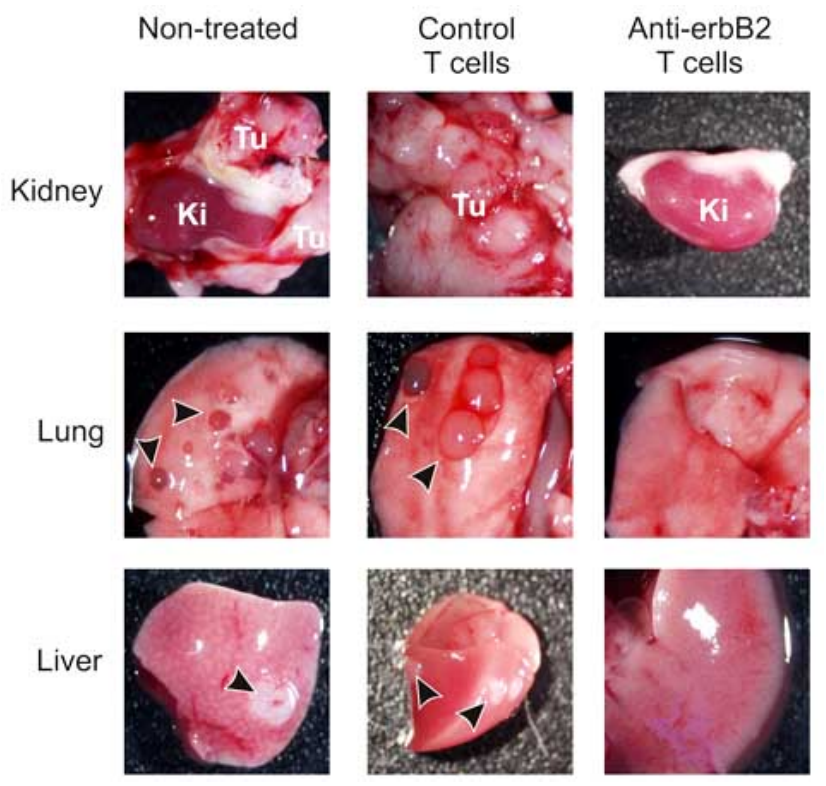

Fig. (6). Anti-erbB2 T cell-treated have a much reduced primary and metastatic tumor burden than control-treated mice. BALB/c-SCID mice were injected beneath the kidney capsule with Renca-erbB2 cells, and were either left untreated, or received an i.v. injection of anti-erbB2 $\mathrm{T}$ cells or control $\mathrm{T}$ cells 1 day later. Photographs of tissues taken on day 21 after tumor injection are shown as listed. Large primary tumors were observed on kidneys of non-treated and control-treated mice, but kidneys of mice receiving anti-erbB2 $\mathrm{T}$ cells were tumor free (some fatty tissue can be seen attached to this kidney). Tumor metastases were also apparent on lungs and liver of non-treated mice, and controltreated mice, whereas no tumor was visible on lung or liver of anti-erbB2 $\mathrm{T}$ cell-treated mice. Arrowheads point to metastases. Photographs are representative of 5 mice per group. $(\mathrm{Tu}=$ tumor; $\mathrm{Ki}=$ kidney).

Microscopic analysis of tissues from mice culled on day 21 , revealed tumors infiltrating normal kidney and lung tissue of control treated mice (Fig. 7). In contrast, no tumor was present in tissues from the majority of anti-erbB2 $\mathrm{T}$ cell treated mice. Interestingly, there were signs of inflammation in kidney, lungs and liver in mice treated with anti-erbB2 $\mathrm{T}$ cells (Fig. 7). In lungs, there was no tumor, and a perivascular and vascular inflammation. This infiltrate around the blood vessels consisted of mainly activated lymphoid cells, with macrophages and fewer numbers of eosinophils and neutrophils. In the kidneys and livers of erbB2 $\mathrm{T}$ cell treated mice, there was inflammation of similar cellular composition, but to a lesser degree than in lungs. The absence of inflammation in areas of normal tissue in control treated mice suggests that this reaction was associated with the presence of erbB2 $2^{+}$tumor cells in the mouse.
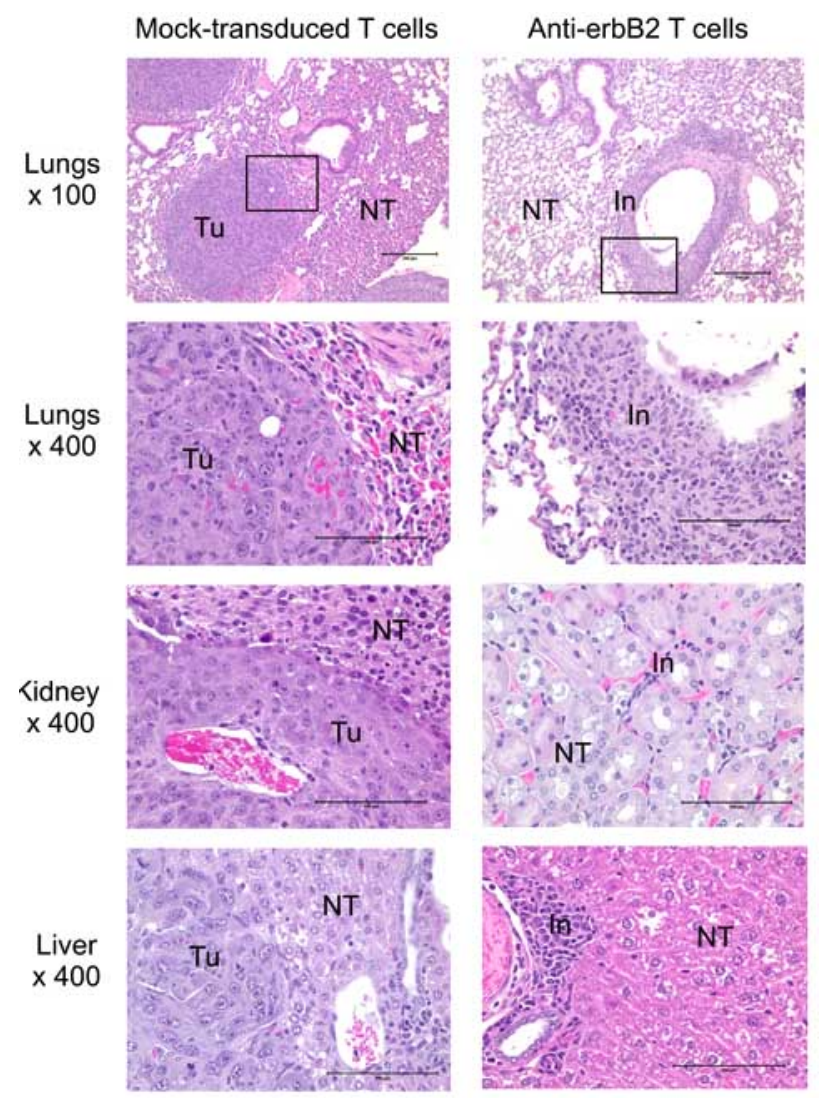

Fig. (7). Tissues from mice injected with anti-erbB2 $T$ cells have an inflammatory infiltrate. Photographs were taken of $\mathrm{H} \& \mathrm{E}$ stained formalin-fixed sections of lung, kidney and liver tissues collected on day 21 after injection sub-kidney capsule with RencaerbB2, followed on day 1 with i.v. injection of $1 \times 10^{7} \mathrm{~T}$ cells transduced with either anti-erbB2 T-body or mock-transduced. AntierbB2 $\mathrm{T}$ cell panels represent sections of tissues from three mice. Non-transduced $\mathrm{T}$ cell panels are representative of two mice. $(\mathrm{Tu}=$ tumor, NT = normal tissue, In = infiltrate).

\section{DISCUSSION}

In this study, we described a spontaneously metastatic orthotopic model of kidney cancer in mice using a transplantable murine renal cell carcinoma expressing the human TAA, erbB2. We demonstrated specific immune targeting of this tumor using mouse $\mathrm{T}$ cells genetically engineered to express an anti-erbB2 T-body, and the eradication of orthotopic RCC by adoptive transfer of genetically engineered $\mathrm{T}$ cells. Adoptive immunotherapy represents a potentially effective cancer treatment option in which tumor-specific $T$ cells are activated and expanded to large numbers before systemic delivery to patients. This process circumvents diffi- 
culties in adequately expanding $\mathrm{T}$ cells in vivo with vaccines, and results in sufficient activation of $\mathrm{T}$ cells away from the frequent immune regulatory factors that can often restrict activation of $\mathrm{T}$ cells, particularly against self antigens typical of many TAA.

The scarcity of endogenous highly tumor-reactive $\mathrm{T}$ cells for most malignancies has led to the use of genetic modification to generate specific T cells $[19,20]$ reactive against cancers originating from many tissues. Kidney cancer is included in the list of tumors targeted, where human $\mathrm{T}$ cells were genetically redirected to respond against renal cell carcinoma cells expressing carbonic anhydrase IX recognized by the G250 monoclonal antibody. Following these in vitro studies, a Phase I study was initiated using adoptive transfer of gene-modified autologous $\mathrm{T}$ cells specific for CA IX for the treatment of renal cell carcinoma in three patients [21]. No anti-tumor responses were documented, and the treatment was associated with some liver toxicity, reaching grade IV in one patient.

Reasoning that research into this approach would be facilitated by the development of a relevant mouse tumor model, we generated an erbB2-expressing mouse RCC cell line and injected it directly into the kidneys of SCID mice. Immunodeficient mice were used in order to assess the effectiveness of gene-modified $\mathrm{T}$ cells in the absence of potential contributions from endogenous anti-erbB2 adaptive immunity. ErbB2-reactive T cells were then generated by genetic modification and adoptively transferred into tumor-bearing mice. Mouse $\mathrm{T}$ cells were used in preference to human $\mathrm{T}$ cells because mouse $\mathrm{T}$ cells have the ability to migrate correctly and persist in mice whereas human $\mathrm{T}$ cells do not. Here we show, for the first time, that mouse $\mathrm{T}$ cells reactive with erbB2 can be generated and used effectively against orthotopic kidney cancer in mice. Some non-specific activity of adoptively transferred $\mathrm{T}$ cells was observed against the erbB2-transduced tumors in vivo, which inhibited tumors. It is not clear what the mechanism of this effect was at this time, although there could be contributions from lymphokine-activated killer (LAK) cell effects. LAK activity has previously been shown to mediated anti-tumor activity [22]. Another potential contribution to the activity of control $\mathrm{T}$ cells could come from an MHC-restricted $\mathrm{T}$ cell response against the human erbB2 antigen. Since mice are not immunologically tolerant of human erbB-2, transfer of low numbers of MHC-restricted erbB-2-specific $\mathrm{T}$ cell precursors may have led to some anti-tumor activity. In addition, some reactivity of erbB-2 transduced $\mathrm{T}$ cells was observed against the erbB-2 negative parental Renca cell line. This activity was significantly lower than that against erbB-2 positive Renca cells $(\mathrm{P} 2=0.008)$, and although the reason is not clear at present, it could have been mediated by non-erbB-2-specific interaction between unknown tumor target molecules and receptors on transduced $\mathrm{T}$ cells. Interactions with adhesion molecules or stress-inducible ligands have previously been demonstrated to induce some function from $T$ cells in the absence of interaction with cognate antigen [23-25].

The presence of leukocytes and reactive appearance of lungs and liver in treated mice, the chief metastatic sites in this model, suggests that adoptively transferred genemodified $\mathrm{T}$ cells are able to mediate changes in the molecular and cellular microenvironment of these tissues, which may inhibit seeding or growth of metastases. It is likely that this response is specifically induced by erbB2 antigen since this inflammatory response was not seen in mice receiving control T cells.

When considering the translational significance of this study, it is important to consider that mice do not express this tumor antigen in any normal tissues. In contrast, humans express erbB-2 in several normal tissues, albeit at lower levels than tumors, and activity of transferred $\mathrm{T}$ cells against normal cells is possible [26, 27]. The absence of Her-2 in mice precludes any assessment of on target toxicity against these normal tissues that could lead to autoimmunity.

Interestingly, in this model, systemically delivered activated $\mathrm{T}$ cells could impact on RCC cells deposited in the kidney, demonstrating their ability to retain the ability to migrate despite in vitro culture. The activated, gene-modified $\mathrm{T}$ cells were able to eradicate tumor cells at this site. In this study, T cells were used against early stage disease, ostensibly a tumor of approximately 30,000 cells, and significant anti-tumor activity observed. Having established the model system, it will now be of interest to determine if improvements can be afforded to therapy by repeated administration of $\mathrm{T}$ cells, or combining adoptive transfer with cytokines that can support $\mathrm{T}$ cells, e.g. the growth factors IL-2 or IL-15. Combining cytokine support or adjuvants with specific immune targeting using adoptively transferred $\mathrm{T}$ cells may lead to enhanced cancer control.

\section{ACKNOWLEDGEMENTS}

This work was supported by grants from The National Health and Medical Research Council of Australia (NHMRC), The Cancer Council of Australia, The Bob Parker Memorial Trust and the Peter MacCallum Cancer Centre Foundation. M.K. is supported by a Senior Research Fellowship from the NHMRC. P.D. is supported by an NHMRC Career Development Award, YX is supported by an NHMRC Peter Doherty Postdoctoral Fellowship and M.J.S. is supported by a Research Fellowship from the NHMRC. We would like to acknowledge the help of Winfried Wels (Chemotherapeutisches Forschungs-institut Georg-Speyer-Haus, Frankfurt am Main, Germay) for supplying the erbB2 single chain chimeric receptor, and Naomi Peter (Peter MacCallum Cancer Centre, Melbourne, Australia) for H\&E staining of tissues sections.

\section{REFERENCES}

[1] Yang JC, Childs R. Immunotherapy for renal cell cancer. J Clin Oncol 2006; 24: 5576-83.

[2] Motzer RJ, Murphy BA, Bacik J, et al. Phase III trial of interferon alfa-2a with or without 13-cis-retinoic acid for patients with advanced renal cell carcinoma. J Clin Oncol 2000; 18: 2972-80.

[3] Rini BI, Zimmerman T, Stadler WM, Gajewski TF, Vogelzang NJ. Allogeneic stem-cell transplantation of renal cell cancer after nonmyeloablative chemotherapy: feasibility, engraftment, and clinical results. J Clin Oncol 2002; 20: 2017-24.

[4] Komohara Y, Harada M, Arima Y, et al. Identification of target antigens in specific immunotherapy for renal cell carcinoma. J Urol 2007; 177: 1157-62.

[5] Pinthus JH, Waks T, Malina V, et al. Adoptive immunotherapy of prostate cancer bone lesions using redirected effector lymphocytes. J Clin Invest 2004; 114 : 1774-81.

[6] Kershaw MH, Westwood JA, Hwu P. Dual-specific T cells combine proliferation and antitumor activity. Nat Biotechnol 2002; 20: 1221-7. 
[7] Brentjens RJ, Latouche JB, Santos E, et al. Eradication of systemic B-cell tumors by genetically targeted human $\mathrm{T}$ lymphocytes co-stimulated by CD80 and interleukin-15. Nat Med 2003; 9: 27986.

[8] Cheadle EJ, Gilham DE, Thistlethwaite FC, Radford JA, Hawkins RE. Killing of non-Hodgkin lymphoma cells by autologous CD19 engineered T cells. Br J Haematol 2005; 129: 322-32.

[9] Maher J, Brentjens RJ, Gunset G, Riviere I, Sadelain M. Human T-lymphocyte cytotoxicity and proliferation directed by a single chimeric TCRzeta /CD28 receptor. Nat Biotechnol 2002; 20: 70-5.

[10] Kahlon KS, Brown C, Cooper LJ, Raubitschek A, Forman SJ, Jensen MC. Specific recognition and killing of glioblastoma multiforme by interleukin 13-zetakine redirected cytolytic $\mathrm{T}$ cells. Cancer Res 2004; 64: 9160-6.

[11] Hombach A, Schlimper C, Sievers E, et al. A recombinant anticarcinoembryonic antigen immunoreceptor with combined CD3zeta-CD28 signalling targets T cells from colorectal cancer patients against their tumour cells. Gut 2006; 55: 1156-64.

[12] Westwood JA, Smyth MJ, Teng MW, et al. Adoptive transfer of T cells modified with a humanized chimeric receptor gene inhibits growth of Lewis-Y-expressing tumors in mice. Proc Natl Acad Sci USA 2005; 102: 19051-6.

[13] Oosterwijk E, Ruiter DJ, Hoedemaeker PJ, et al. Monoclonal antibody G 250 recognizes a determinant present in renal-cell carcinoma and absent from normal kidney. Int J Cancer 1986; 38: 489-94.

[14] Seliger B, Rongcun Y, Atkins D, et al. HER-2/neu is expressed in human renal cell carcinoma at heterogeneous levels independently of tumor grading and staging and can be recognized by HLA-A2.1restricted cytotoxic T lymphocytes. Int J Cancer 2000; 87: 349-59.

[15] Lamers CH, Langeveld SC, Groot-van Ruijven CM, Debets R, Sleijfer S, Gratama JW. Gene-modified T cells for adoptive immunotherapy of renal cell cancer maintain transgene-specific immune functions in vivo. Cancer Immunol Immunother 2007.

[16] Huben RP, Connelly R, Goldrosen MH, Murphy GP, Pontes JE. Immunotherapy of a murine renal cancer. J Urol 1983; 129: 10758.
[17] Darcy PK, Haynes NM, Snook MB, et al. Redirected perforindependent lysis of colon carcinoma by ex vivo genetically engineered CTL. J Immunol 2000; 164: 3705-12.

[18] Haynes NM, Trapani JA, Teng MW, et al. Single-chain antigen recognition receptors that costimulate potent rejection of established experimental tumors. Blood 2002; 100: 3155-63.

[19] Ho WY, Blattman JN, Dossett ML, Yee C, Greenberg PD. Adoptive immunotherapy: engineering $\mathrm{T}$ cell responses as biologic weapons for tumor mass destruction. Cancer Cell 2003; 3: 431-7.

[20] Kershaw MH, Teng MW, Smyth MJ, Darcy PK. Supernatural T cells: genetic modification of $\mathrm{T}$ cells for cancer therapy. Nat Rev Immunol 2005; 5: 928-40.

[21] Lamers CH, Sleijfer S, Willemsen RA, et al. Adoptive immunogene therapy of cancer with single chain antibody $[\mathrm{scFv}(\mathrm{Ig})]$ gene modified T lymphocytes. J Biol Regul Homeost Agents 2004; 18: 134-40.

[22] Grimm EA, Robb RJ, Roth JA, et al. Lymphokine-activated killer cell phenomenon. III. Evidence that IL-2 is sufficient for direct activation of peripheral blood lymphocytes into lymphokine-activated killer cells. J Exp Med 1983; 158: 1356-61.

[23] Li YQ, Kobayashi M, Yuan L, et al. Protein kinase C mediates the signal for interferon-gamma mRNA expression in cytotoxic T cells after their adhesion to laminin. Immunology 1998; 93: 455-61.

[24] Takahashi K, Nakamura T, Adachi H, Yagita H, Okumura K. Antigen-independent $\mathrm{T}$ cell activation mediated by a very late activation antigen-like extracellular matrix receptor. Eur $\mathrm{J}$ Immunol 1991; 21: 1559-62.

[25] Bauer S, Groh V, Wu J, et al. Activation of NK cells and T cells by NKG2D, a receptor for stress-inducible MICA. Science 1999; 285 727-9.

[26] Coussens L, Yang-Feng TL, Liao YC, et al. Tyrosine kinase receptor with extensive homology to EGF receptor shares chromosomal location with neu oncogene. Science 1985; 230: 1132-9.

[27] Weiner DB, Nordberg J, Robinson R, et al. Expression of the neu gene-encoded protein (P185neu) in human non-small cell carcinomas of the lung. Cancer Res 1990; 50: 421-5. 